\title{
INTERPRETACIÓN DE LOS PAISAJES AGRARIOS DE LA TOSCANA A FINES DEL SIGLO XVIII POR EL ILUSTRADO G.B LANDESCHI ${ }^{1}$
}

\author{
María Hernández Hernández
}

\begin{abstract}
RESUMEN
A finales de la centuria ilustrada en amplios sectores de la Toscana se observan claros indicios de degradación ambiental (disminución de la superficie arbolada, intensificación de los procesos de pérdida de suelos en oteros y colinas, etc.) que se tradujeron en la aparición de una nueva postura ante el medio natural (política de plantíos, nueva ordenación de los espacios agrarios). Afirmaciones que pueden sintetizarse en unos principios, (armonización del desarrollo económico y protección de la naturaleza o defensa de los usos potenciales del suelo), que gozan de una gran vigencia en la actualidad. Principios que aparecen claramente reflejados en Saggi di Agricoltura.
\end{abstract}

\begin{abstract}
At the end of the 17th century in wide spaces of Toscana, it is shown obvious evidences of environment degradation (the decreasing of the wooden area or become more intensive soil lost in hillocks and hills). Fact which is expressed in the appearance of a new point of view about environment (planted ground policy, new agrarian space management) that can be reduced to some principles: arrange economic development and nature defence or soil potential usage protection. Ideas which have a remarkable use at the present time and appear clearly revealed in th Saggi di Agricoltura.
\end{abstract}

\section{Introducción}

La irregular distribución pluviométrica, las condiciones litológicas, la evolución histórica, la estructura social, etc. han condicionado desde la antigüedad los usos del suelo y en particular la agricultura. De modo que las actividades antrópicas se han caracterizado por

1 Este estudio forma parte de la memoria de licenciatura realizada por la misma autora con el título «Interpretación de los paisajes agrarios de la Toscana y Valencia en el siglo XVIII vistos por Landeschi y Cavanilles: paralelismos e influencias». 
una intervención secular para adaptarse a las condiciones de semiaridez imperantes o para intentar minimizar los procesos erosivos. Intervencionismo que en la mayoría de las ocasiones supondrá una modificación relevante del paisaje natural, el cual era poco proclive a los intereses de sus moradores.

Adaptación al medio que se obtiene mediante una serie de técnicas (abancalamiento de terrazas, derivación de aguas, etc.) que tienen un gran significado desde el punto de vista medio ambiental y cultural. Valor este último que debemos relacionar con la existencia de una serie de sociedades agrarias de subsistencia que a lo largo de sucesivas generaciones han ido desarrollando y mejorando sus conocimientos agronómicos. Desde el punto de vista medioambiental, su relevancia se vincula con el intento de minimizar los efectos de las aguas de arroyada al laminar las crecidas al distribuir el agua entre las diversas terrazas.

Aumento de estos procesos que hay que relacionar con la desorganización que se produce a partir de los años 60 cuando estas zonas debido a sus características definidoras (reducida superficie y el alto valor de la pendiente) no pueden ser competitivas en la economía de mercado que se instauraba en España o con el potenciamiento de la denominada Italia Central o Terza Regione. Sin embargo, su intensificación no es un fenómeno que debamos restringir a la segunda mitad del siglo XX sino que en la centuria ilustrada, ya se manifiestan con gran virulencia como confirma la lectura de numerosas obras de este período. Situación que será denunciada tanto a nivel individual (como pueden ser las obras de Landeschi o Cavanilles) como colectivo (Academia dei Georgofili o Sociedades de Amigos del País) quienes propugnarán una nueva ordenación de los espacios agrarios, sustitución de los sistemas de tenencia, etc. para intentar reducir los efectos ocasionados por una inadecuada dirección de las aguas, pero también para aumentar los rendimientos agrícolas en una época en que una mayor presión demográfica exigía un incremento en las disponibilidades de alimentos.

\section{Significación y relevancia de G. B. Landeschi}

Su trascendencia puede evidenciarse haciendo referencia a la impronta que su obra (Saggi di Agricoltura, publicado hacia 1770) ha tenido en la sistematización de las laderas o en la defensa de los postulados ilustrados. Sin embargo, también hemos de manifestar que es un autor prácticamente desconocido en la Geografía española aunque no por ello carente de importancia y significación para aprehender la impronta que las actividades antrópicas causan en un entorno natural y las posibles actuaciones a adoptar para intentar reducir las diversas manifestaciones de degradación ambiental que éstas pudieran ocasionar.

\subsection{Landeschi, testimonio de una naciente inquietud por la degradación ambiental}

Existen motivos, tal como indica Urteaga ${ }^{2}$, para pensar que hasta el siglo XVIII la cultura europea estuvo lejos de formular un cuerpo razonable de ideas conservacionistas. Pese a que algún autor ha creído ver en el arbitrista español Caxa de Leruela un «precursor de la observación ecológica» y que, del mismo modo, podrían encontrarse aquí y allá observaciones marginales y notas secundarias que nos hablan del interés por la protección del medio natural en algunos autores anteriores del siglo XVII, hasta la centuria ilustrada

2 URTEAGA, U.: La tierra esquilmada, Serba-CSIC, Madrid, 1987, p. 15. 
existen barreras ${ }^{3}$ que dificultan notablemente la construcción de una doctrina coherente sobre la conservación del medio físico. La Ilustración reconsiderará el triángulo DiosHombre-Naturaleza desde una nueva perspectiva. Pocos negarán la existencia divina y su impulso creador, pero el papel que en la tradición jugaba la Providencia pasará a ser ocupado por la Razón.

El conservacionismo, tal como puede entenderse en la centuria ilustrada, supone, en consecuencia, un desarrollo complementario de diferentes nociones científicas. Primero, la idea de que los recursos son limitados, de ahí la necesidad de una explotación cautelosa de la naturaleza. Segundo, la percepción bastante clara de los lazos que vinculan a los organismos entre sí y a éstos con el medio ambiente físico (atmosférico, edafológico y botánico). Tercero, la noción de la fragilidad del equilibrio natural. Estabilidad que puede verse alterada por la acción sobreexplotadora de la sociedad, deduciéndose de los elementos anteriormente indicados la necesidad de defender el «orden natural». Relacionado con la afirmación precedente, está la evidencia de la capacidad depredadora del hombre y su gran potencial destructivo, que puede provocar un declive de los recursos naturales. Cuarto, y en el terreno económico, se manifiesta una clara condena del despilfarro en la administración de éstos y se evidencia la necesidad de una acción cautelar, incluso mediante leyes, que favorezca la protección y conservación de la naturaleza.

\subsubsection{Defensa de una nueva sistematización agraria}

La sistematización de las laderas será, por tanto, reflejo de una concienciación creciente ante las manifestaciones de una degradación ambiental cada vez más evidente. La preocupación por estos síntomas (pérdidas en el horizonte edáfico, disminución de la superficie arbolada, etc.) aparece recogida en numerosas obras del período analizado.

Problema cuyo origen es antiguo (en 1733 el porcentaje de terrenos afectados por procesos erosivos suponía el $11 \%$ de la superficie de las Colinas de Asisi ${ }^{4}$ ) y que se relaciona con el sistema de cultivo tradicional practicado ${ }^{5}$, pero que se acrecienta al ampliarse la superficie cultivada y el asentamiento de nuevos colonos.

Frente al sistema de cultivo tradicional, la Academia dei Georgofili propone una nueva sistematización de los paisajes agrarios. Ordenación en la que el tema del agua es una constante pues unos adecuados conocimientos sobre su gestión es fundamental gracias a los beneficios que éstas reportan a las haciendas (aporte de limo, de tierra nueva, etc.) frente a los aspectos negativos que las turbias originan cuando se abandona esta sistematización (incremento de las pérdidas de suelo).

3 La principal de estas barreras era la pervivencia de una explicación providencial del mundo: en la cultura cristiana la lógica de los seres se encuentra en última instancia en el creador. Aceptada esta afirmación la preservación del mundo correspondía a Dios. De ahí que la responsabilidad del hombre ante el mundo físico es algo bastante secundario, en tanto que el orden de los seres vivos viene garantizado por el orden divino.

4 DESPLANQUES, H.: «I paesaggi collinari tosco-umbro-marchigiani» I paessagi umani, Touring Club italiano, Turín, 1977, p. 104.

5 Sistema de cultivo tradicional (rittochino) que se caracteriza por cultivar siguiendo las líneas de máxima pendiente para favorecer el drenaje de unos espacios en los que debido a las litologías dominantes (impermeables y fácilmente erosionables) y la existencia de una orografía intrincada, la evacuación de las aguas era el objetivo prioritario, aun cuando se ocasionase pérdidas edáficas. 


\subsubsection{Defensa de la superficie arbolada}

El árbol (su defensa, conocimiento y propagación) será una de las inquietudes de estos pensadores e incluso podemos afirmar que «la filosofía forestal ilustrada puede, quizás, erigirse en uno de los símbolos de las muchas novedades positivas aportadas por el espíritu ilustrado sobre la naturaleza en general y sobre los recursos naturales en particular» ${ }^{6}$.

El clamor de los ilustrados en apoyo del árbol nace, como es lógico, de un hecho visual como era la apreciación de una clara y progresiva deforestación. Proceso que hay que relacionar con una serie de causas entre las que hay que distinguir entre las técnicas (aumento de la demanda de madera, roturación de nuevas tierras debido a la mayor presión demográfica) y las legislativas o administrativas (política de plantíos).

Si la política forestal del despotismo ilustrado ofrece un balance bastante decepcionante en sus realizaciones, no podemos decir lo mismo de la reflexión de los ilustrados sobre el bosque. Así, podemos afirmar que aunque el objetivo de proteger la riqueza forestal no se logró en el siglo XVIII, al menos, se pusieron las bases de un discurso científico. En sus escritos se abre paso la idea de que los bosques en España, en Toscana son un recurso escaso, expoliado en demasía y difícilmente renovable. Por ello, una y otra vez insistirán en la necesidad de una explotación cuidadosa de las masas forestales. Gestión racional que exigía, en primer término, desterrar algunos prejuicios ancestrales ${ }^{7}$ y promover un conocimiento científico del arbolado. En suma, una tarea pedagógica de difusión de la arboricultura y la silvicultura que los ilustrados emprenderán con el mismo empeño desplegado en la promoción de cualquier «ciencia útil».

Bosques que generan una serie de beneficios económicos, pero tienen también un gran significado desde el punto de vista medioambiental ya que contribuyen a detener los procesos de pérdida de suelos pues «encontrándose, por lo general, los bosques en lugares escarpados y donde caen y discurren las aguas de lluvia, allí los matorrales al endurecer el terreno con sus raíces, impiden los desmoronamientos y las roturas y de este modo se conservan más fácilmente las terrazas superiores cultivadas y los valles no son tan hendidos por el agua» ${ }^{8}$.

\subsubsection{Mantenimiento de los usos potenciales del suelo}

Organización de los espacios agrarios que alberga un concepto inherente como es la defensa de la naturaleza. Landeschi o Cavanilles, como reflejo de un movimiento más amplio, no dudan en afirmar que ésta no se debe forzar. Postura que ratifican al aseverar que no se deben poner en cultivo aquellas tierras que por sus condiciones topográficas (abruptas, elevadas pendientes o terrazas con escaso horizonte edáfico) tradicionalmente se han dedicado a pastos o a bosques. Usos que, como el propio ilustrado toscano indica, son también necesarios ya que proporcionan alimento para el ganado y éste suministra el

6 LÓPEZ LINAJE, J.: Agricultores, botánicos y manufactureros en el siglo XVIII, coedit MAPA-BCA, Madrid 1989, p. 95.

7 Prejuicios ancestrales que aparecen recogidos en la obra de Landeschi quien rechaza afirmaciones como «los bosques son un obstáculo para la agricultura» y que sirven de justificación a la tala de muchos bosques y la dedicación de antiguas superficies boscosas para tierras de cultivo. Otros se relacionaban, en cambio, con el problema de la fertilidad de los campos. Así muchos campesinos pensaban que la existencia de árboles incluso en los linderos de los campos de cultivo perjudicaba a las cosechas, se decía que la vegetación arbórea «robaba sustancia» a la tierra y también la sombra de los árboles impedía el crecimiento natural de los sembrados.

8 LANDESCHI, G.B.: Saggi di Agricoltura, ed. correta e aumentata da A. Bicchi, Florencia, 1807, capítulo XLVII, parte segunda, 187. 
estiércol tan necesario para devolver la fertilidad a la tierra. Cambio en el uso del suelo que conllevará la intensificación de los procesos erosivos (acarcavamiento, pérdida de suelo, etc.).

Deterioro, por tanto, que debemos relacionar con la introducción de unos usos (puesta en cultivo de los valles y el abandono progresivo de las heredades en colinas y oteros), con la desaparición de unas prácticas tradicionales (descuido de las labores que aseguraban la conservación de la sistematización colinar) que supera su capacidad de acogida o con el cambio de costumbres como confirma la afirmación siguiente: «en los siglos pasados las colinas y los oteros tenían una gran producción y ahora son casi estériles es que entonces se cultivaban y se trabajaban por los mismos propietarios que habitaban allí mismo, los cuales al trabajar y cultivarlos no tenían otro objetivo que el de obtener de la tierra algún haz de más al año y no sufrir pocas fatigas como hacen nuestros aparceros. Su mayor interés era, por tanto, mantener los fondos productivos y aumentar los ingresos para sus familias en el futuro. En cambio, son muy diferentes los miserables apareceros de los tiempos actuales los cuales no piensan nada en el futuro, ni en los daños venideros, anteponiendo unos pocos haces de trigo a la ruina de una parcela, que puede derivarse al deshacer un talud, es decir, eligen lo poco presente a lo mucho futuro»?

\subsection{Landeschi, ejemplo de una creciente preocupación social}

Manuscrito en el que además de la ya citada preocupación por la degradación medioambiental, también aparecen una notable inquietud por la estructura social.

En una sociedad agraria profundamente ajena a la cultura, con porcentajes muy altos de analfabetismo, sin enseñanza primaria, fuera de la que impartían instituciones privadas como conventos y algunas Sociedades Económicas, el sacerdote resultaba un «vehículo» nada despreciable para influir en ella. Al menos teóricamente disponía de una cultura que le capacitaba para comprender la información que se le suministraba y vulgarizarla. Además, su mismo ministerio le dotaba de una posición moralmente prestigiosa en el municipio o aldea rural con la que su influencia beneficiosa se veía fortalecida, pues «nadie mejor que los párrocos conocerían la situación de sus feligreses, su tipo de vida y sus necesidades» ${ }^{10}$.

Así, Landeschi ${ }^{11}$ en Saggi di Agricoltura indica que es muy necesario que los párrocos cultiven sus haciendas pues de este modo conseguirán aumentar la producción de sus tierras y podrán, en consecuencia, entregar cuantiosas limosnas a los más necesitados y no depender de las ofrendas recogidas en las celebraciones litúrgicas para sobrevivir y, por tanto, acrecentar las cargas que deben tolerar los pobres campesinos con lo que se atraerán el apoyo y el aprecio de sus feligreses. Afirmaciones que debemos relacionar con los nuevas corrientes (regalismo, jansenismo) que se difunden y con las que se pretende que la iglesia retorne a sus orígenes (importancia de los párrocos frente al clero regular, lectura de la Biblia, lucha contra la superchería, etc.). Principios que son compartidos y defendidos por el ilustrado toscano.

Sin embargo, a pesar de la relevancia que concede a los reverendos, no duda en criticar la situación parasitaria de la mayor parte del clero (no sólo los beneficiarios, sino incluso el regular) quienes no tenían ningún tipo de actividad, ni siquiera pastoral, pues únicamente

9 Ibíd., capítulo XVI, primera parte, p. 55.

10 CARANDE, R.: «El despotismo ilustrado de los Amigos del País», Siete estudios de Historia de España, Ariel, Esplugues, p. 176.

11 La relevancia que el ilustrado toscano concede a los párrocos en relación a la difusión de los nuevos conocimientos agronómicos aparece recogido en los capítulos XXI, XXIII, XXVII, XXVIII y XXIX de la primera parte de Saggi di Agricoltura. 
una mínima parte atendía directamente y con exclusividad la dirección espiritual del pueblo.

Debemos, asimismo, destacar la defensa de amplios sectores de la sociedad, generalmente los más desfavorecidos, pero que son los generadores de la riqueza. Preocupación que se traduce en el hecho de que Landeschi, dedique la primera parte de su obra a indicar toda una serie de disposiciones que deben adoptar los propietarios al administrar sus propiedades (introducción de manufacturas, cría de gusanos de seda, adelanto de víveres, aperos, mejoras en los cultivos y en las técnicas, etc.) para evitar su endeudamiento. Se propugna, por tanto, un nuevo sistema de gestión de las haciendas en el que se prioriza la obtención de beneficios frente a la época feudal en que las tierras constituían más un elemento de prestigio social que de riqueza en sí mismas.

Junto a este modelo de administración, los ilustrados defienden el arrendamiento e incluso la enfiteusis a fin de asegurar la posesión de una parcela por un período largo y así muchos relacionan la prosperidad de Cataluña y Valencia con estos sistemas frente a la aparcería a la cual acusan, junto al absentismo, de la decadencia en que se encuentra sumido el agro. Difusión de nuevos sistemas de tenencia con los que se intenta solventar uno de los principales problemas que enfrentaba tanto la agricultura valenciana como la toscana como era el significativo porcentaje de tierras que estaba en manos muertas o sometidas a mayorazgos. Situación que apartaba del mercado de la tierra una considerable superficie agrícola lo que dificultaba su adquisición por aquéllos que querían llevar adelante una explotación con criterios más racionales.

Sistema de tenencia (arrendamiento) que se irá extendiendo durante el siglo XVIII y que contribuirá a crear una distinción entre aquellos propietarios que continuaron manteniendo el sistema de aparcería y, en consecuencia, necesitaban el predio para mantener su renta y aquéllos que la obtendrán a través de su arrendamiento lo que se traducirá en una renta variable y el inicio de una economía de signo capitalista en la cual prima la producción y en donde el trabajo humano va convirtiéndose en una mercancía al quedar desvinculado el campesinado de sus medios de producción (tierra) ${ }^{12}$.

Arrendamientos que deben cumplir una serie de condiciones mínimas (si se desean lograr los nuevos objetivos de justicia social que se pretenden) entre las que destaca: ser a largo plazo (alrededor de treinta años como se indica en el libro Saggi di Agricoltura) para dar seguridad a los campesino que las cultivan o convertirlos, en palabras del propio Landeschi, en «casi propietarios». Evitando de este modo algunos de los aspectos negativos entre los que destacan: cultivar pensando únicamente en obtener los mayores beneficios posibles, entregar las tierras en arrendamiento a los mismos campesinos que las cultivan, rechazando concederlas a aquéllos que ofrezcan un elevado canon ya que las sobrexplotarán para intentar cubrir el desembolso inicial que realizaron y, al igual que los arrendadores a corto plazo y los aparceros, descuidarán todas las labores de conservación, de mantenimiento tan necesarias para asegurar la productividad de las haciendas ubicadas en oteros y colinas.

Sin embargo, la sustitución de la aparcería por el arriendo a largo plazo justificado por el convencimiento de que con tal contrato el predio será mejorado es incluso criticado por Bicchi ${ }^{13}$ quien en el prólogo de la segunda edición manifiesta que «las razones que aduce Landeschi no tienen base suficiente para creer en la superioridad del arriendo» ${ }^{14}$. Afirmación con la que se desanima cualquier intento de modificación contractual.

12 KRIEDTE, P.: Feudalismo tardío y capitalismo mercantil, Crítica, Barcelona, 1991, p. 145.

13 Miembro de la Academia dei Georgofili que redactó e incluyó nuevas notas en la segunda edición de la obra de Landeschi.

14 LANDESCHI, G.B.: op. cit., capítulo XVII, nota 19, p. 57. 
Junto a la defensa del arrendamiento como sistema de tenencia, debemos también indicar que desde la concepción ilustrada se propugna una concentración parcelaria y la posterior asignación de lotes de tierra adecuados a las posibilidades reales de los campesinos. Con este último aspecto se pretende, además, desterrar un prejuicio muy extendido en esta época como es considerar que superficie cultivada y producción son dos variables proporcionales. Axioma que es rechazado por el iluminado toscano quien afirma que «se mantendrá la superficie cultivada en proporción al tamaño de la familia y el número de bestias aun cuando los campesinos estén siempre ávidos de anexionar nuevas parcelas a su predio debido a su corta inteligencia» pues «la productividad de la tierra depende mucho más de la diligencia del hombre al cultivarla que de la propia tierra ${ }^{15}$.

Concentración parcelaria que hay que relacionar con la crítica que se realiza a la aparcería como sistema de tenencia. Sistema y régimen de tenencia propio de una economía cerrada que justifica que en todos los predios se practique la policultura para lograr el autoabastecimiento y junto a éste, una dispersión parcelaria para, de este modo, disponer de parcelas de diversa naturaleza (aptas para prados, para el cultivo de cereales, vid, etc.). En consecuencia, la sustitución de la aparecería y la reorganización parcelaria serían dos manifestaciones de un mismo fenómeno: la racionalización de los paisajes agrarios. Intento de modernizar la estructura estatal en el que tiene una gran relevancia las nuevas teorías económicas (fisiocracia) que se manifestarán en el deseo de abolir, transformar todos aquellos aspectos que se oponen a un desarrollo de las actividades económicas.

\subsection{Divulgación de los principios ilustrados}

Hemos asimismo de hacer referencia al papel que Landeschi y su obra juega en la difusión de los principios fisiocráticos e ilustrados, pues no hemos de olvidar que uno de los objetivos de estos pensadores tanto a nivel individual como colectivo (Academia dei Georgofili, Sociedades de Amigos del País) será hacerla progresar con el empleo de nuevas técnicas, con la crítica de aquéllas que sean inadecuadas, etc.

Durante el Antiguo Régimen el único medio factible de acrecentar la producción era roturar nuevas tierras debido a la escasez de medios técnicos de los que se disponían. Frente al sistema cerealícola «de año y vez»o «al tercio» dominante en amplias zonas durante gran parte del siglo XVIII, los autores que tratan la agricultura en este período (sobre todo en la segunda mitad de la centuria), comienzan a propugnar una intensificación de los cultivos introduciendo nuevas técnicas entre las que destacan la sustitución del barbecho con la rotación de cultivos utilizando legumbres (guisantes, habas), forrajes (trébol y altramuz). Sistema con el que se postulaba la integración de la agricultura y la ganadería al alternarse pastos y cultivos y se posibilitaba el aumento de la cabaña ganadera gracias a la ampliación de los forrajes lo que se tradujo, asimismo, en el incremento de la cantidad de estiércol disponible tan necesario para devolver la fertilidad a las tierras cultivadas. Ejemplo de estas novedades es la introducción del cultivo de la alholva en las parcelas que debían dedicarse a barbecho. Rotación que es descrita en la nota 24 de Saggi di Agricoltura.

No obstante, el fracaso contemporáneo de los esfuerzos ilustrados por introducir una nueva agricultura es incuestionable. Ineficacia que hay que relacionar al menos con cinco grandes causas, una de ellas de carácter físico y el resto sociales. La primera explicación debe referirse al medio físico, y dentro de él señaladamente al régimen de lluvias, tempe-

15 Ibíd, capítulo XX, primera parte, pp. 63-64. 
raturas y balance hídrico. Pero, aunque eran importantes las limitaciones del medio físico, todavía lo eran más las que provenían del tipo de usos y costumbres emanadas de una determinada organización social ${ }^{16}$, del tipo de propiedad vigente ${ }^{17}$, del laberíntico sistema impositivo que gravaba las cosechas ${ }^{18}$ y la falta de formación técnica adecuada. Por tanto, en la región levantina o toscana las innovaciones se manifiestan únicamente en un intento de mejorar los cultivos perfeccionando sus técnicas.

Ampliar los conocimientos agrarios y disminuir la incultura entre campesinos y propietarios explica que tanto Cavanilles y, sobre todo, Landeschi describan detalladamente todas las labores, cuidados que requieren los cultivos (poda, época más adecuada para sembrarlos, como y cuando se debe plantar árboles, cuando se deben renovar los plantíos decadentes, etc.) así como indicar que labores, costumbres son inadecuadas y que, por tanto, deben evitarse (por ejemplo, arar las tierras fuera de sazón).

Ignorancia que en muchas ocasiones debemos relacionar con el hecho de que sus conocimientos son transmitidos de generación en generación, pensando que este es el mejor modo de cultivar y negándose a introducir innovaciones e incluso debido a la existencia de juicios preestablecidos, en la mayoría de las ocasiones erróneos y entre los que cabría citar, por ejemplo, que los labradores e incluso los señores piensen que a mayor superficie mayor productividad.

Lucha contra la falta de conocimientos en la que se incluyen toda una serie de disposiciones que postulan por un desarrollo de las ciencias útiles frente al anquilosamiento de los estudios tradicionales ${ }^{19}$. Propugnándose frente a los excesos de la escolástica, una reforma de la enseñanza. Reforma en la que se proponen una serie de alternativas como son: creación de instituciones donde se enseñen las ciencias útiles, con una particular insistencia en el estudio de la agricultura y la introducción de nuevos métodos pedagógicos que acaben con las prácticas tradicionales basadas en burdos principios adoptados sin previa reflexión sobre su efectividad y claramente opresivos para el alumno.

Landeschi propondrá para acabar con esta «impericia» una serie de disposiciones entre las que destacan: «llevar a los colegiales en los días de verano a la campiña para que observen las diversas labores agrícolas, aprendan los oportunos cuidados y al mismo tiempo se ejerciten realizando algunos de las tareas más sencillas (...), cierto es también que deberían observar algunas haciendas trabajadas diligentemente, así como otras dirigidas de manera negligente. Convendría que para obtener el mismo fin, se les indicase el

16 La práctica de la nueva agricultura permitía sostener una cabaña abundante al margen de la utilización de las rastrojeras, y sobre todo, barbecheras. Implica, por tanto, que el campesino puede acotar su parcela al uso ganadero colectivo y establecer sobre ella el sistema o la rotación de cultivos que le parezca más conveniente a sus necesidades.

17 Propiedad que se caracterizaba por el bajo nivel de equipamiento o capitalización ya que tanto el capital invertido en aperos, herramientas, ganado trabajo, edificios, medios de acarreos como a procurarse medios productivos ordinarios (semillas, abonos, etc.) se encontraba por debajo del nivel deseable. La razón estribaba en el carácter temporal y precario que afectaba directamente al usufructo campesino de las tierras y, a veces, ganados otorgados por la propiedad.

18 El sistema impositivo e incluso las disposiciones legislativas restrictivas explican los reducidos estímulos comerciales que tenían las explotaciones: los campesinos únicamente podían aspirar a vender en un mercado muy local la porción de cosecha finalmente disponible, en el caso que existieran excedentes, ya que el comercio a escala mayor les estaba vedado por razones legales primero y, después, por la costos inversión, relaciones y formación necesaria.

19 «No critico que a los jóvenes estudiantes se les haga aprender retórica, geometría, filosofía y otras ciencias; pero, ya que una parte de la verdadera sabiduría consiste en conceder a todas las cosas si justa consideración, convendría que las técnicas agrícolas no fueran ignoradas por los jóvenes estudiantes y que éstos no desconocieran sus ventajas y sus valores ocultos» LANDESCHI, G.B: op. cit., capítulo XII, parte primera, p. 89 . 
nombre de los árboles y sus frutos, que plantas se adaptan mejor a la tierra suelta, cuales requieren el monte ${ }^{20} \gg$.

Otro de los principios de la Ilustración y que aparece relacionado con los apartados anteriormente analizados es el intentar devolver a esta actividad el prestigio perdido. El ilustrado toscano no duda en aseverar que la decadencia en que se encuentra sumida la agricultura en San Miniato se debe a que los propietarios son absentistas quienes, además, desprecian ocuparse de ella.

Frente al desprestigio de la agricultura, ampliamente difundido entre los propietarios tanto civiles como eclesiásticos, manifiesta que es «el arte más necesario para la humanidad, pues ésta suministra todas las materias primas que son necesarias para el mantenimiento de la vida humana o que los propietarios no deben avergonzarse de preocuparse por la gestión de sus tierras, «ni deben aborrecer el nombre de agrícola ya que el mismo hijo de Dios llamó con tal nombre a su Divino padre ${ }^{21} \gg$.

Como consecuencia de las afirmaciones anteriores, no duda en afirmar que la reducción de la producción se debe al cambio de costumbres que se ha producido en la sociedad (absentismo, difusión de la ostentación y de numerosos vicios como son la afición a los juegos, al teatro, etc.).

\section{Bibliografía}

BLANCHEMANCHE, P.: Bâtisseurs de Paysages. Terrassement. Épirrement et petite hydraulique agricoles en Europe XVIIe-XIXe siècles, Editions de la Maisons des ciencies de l'homme, Paris, 1990.

CAROSELLLI, M. R.: Critica alla mezzadria di un vescovo del'700, Milán, 1963.

CARUSO, G.: «I sistemi di amministrazione e la questione sociale», La mezzadria negli scritti dei Georgofili, Bolonia, 1936.

CAVANILLES, A. J.: Observaciones sobre la Historia Natural, Geografía, Agricultura, Población y frutos del Reyno de Valencia, Imprenta Real, Madrid, 1797.

CHIARENTI, F.: Riflessioni e osservazioni sull'agricoltura toscana e particolarmente sull'istituzione de'fattori, sul metodo del Landeschi e sull'ordinamento colonico, Pistoia, 1819.

DESPLANQUES, H.: «I paesaggi collinari tosco-umbro-marchigiani», I paesaggi umani, Touring Club Italiano, Turín, 1977.

DUHAMEL DE MONCEAU, H. L.: Tratado del cuidado y aprovechamiento de los montes y bosques, corta, poda, beneficios y usos de sus maderas y leñas, J. Ibarra, Madrid, 1773-74.

IMBERCIADORI, I.: Mezzadria classica toscana, Florencia, 1951.

LANDESCHI, G. B.: Saggi di agricoltura, Ed. corretta e aumentata da A. Bicchi, Firenze, 1807.

LUZZATTO, G.: «La rinascita agraria in Toscana al tempo dei primi Lorenesi», Storia económica dell'età moderna e contemporanea, Cedam, Padova, 1960.

MIRRI, M.: «Propietari e contadini toscani nelle Riforme leopoldine», Movimento operaio, 1955.

MORALES GIL, A.: «El riego con aguas de avenida en las laderas subáridas», Papeles de Geografía, 1, Murcia, 1969, pp. 167-191.

MORALES GIL, A. y BOX AMORÓS, M.: «El aprovechamiento del agua y los suelos en un dominio semiárido: la cuenca del Barranco Blanco. Agost (Alicante), Investigaciones geográficas, 4, Alicante, 1986, pp. 7-24.

NEBOIT, R.: L'homme et l'erosion, Ass. des Publ. Fac. des lettres et Sciences Humaines de Clermont-Ferrand, 1983.

OLIVA, A.: Le sistemazioni idraulico-agrarie dei terreni asciutti di pianura, collina e montagna, Florencia, 1938.

20 Ibíd., capítulo XXXII, primera parte, pp. 88-90.

21 Ibíd., capítulo XXI. Primera parte, p. 65 y capítulo XXIII, p. 70, respectivamente. 
PASSMORE, J.: La responsabilidad del hombre frente a la naturaleza, Madrid, Alianza Universidad, 1978.

PAZZAGLI, C.: L'agricoltura toscana nella prima metá dell'800, Florencia, 1973.

PÉREZ QUINTERO, M. I.: Pensamientos políticos y económicos dirigidos a promover en España la agricultura y demás industrias, Imprenta D. Benito García de Costra y compañía, Madrid, 1789.

SERENI, E.: Storia del paesaggio agrario italiano, Laterza, Bari, 1962.

TRICART, J. y KILIAN, A.: La eco-geografía y la ordenación del medio natural, François Niasperu, París, 1979.

URTEAGA, U.: La tierra esquilmada (las ideas sobre la conservación de la naturaleza en la cultura española del siglo XVIII, Coedit Serbal-CSIC, Madrid, 1987.

VILÁ VALENTÍ, J.: «L' irrigation per nappes pluviales dans le Sud-Est de l'Espagne», Mediterrannée, II, 1961, pp. 19-31.

WOLF, S. T.: «La storia política e sociale: la ripresa dell'Italia (1700-1759)», Storia d'Italia. vol. III: dal primo settecento all’unitá, Giulio Einaudi Editore, Turín, 1983. 